\title{
A New Nearest Neighbor Rule for Text Categorization
}

\author{
Reynaldo Gil-García and Aurora Pons-Porrata \\ Center of Pattern Recognition and Data Mining \\ Universidad de Oriente, Santiago de Cuba, Cuba \\ \{gil, aurora\}@app.uo.edu.cu
}

\begin{abstract}
The nearest neighbor (NN) rule is usually chosen in a large number of pattern recognition systems due to its simplicity and good properties. In particular, this rule has been successfully applied to text categorization. A vast number of NN algorithms have been developed during the last years. They differ in how they find the nearest neighbors, how they obtain the votes of categories, and which decision rule they use. A new NN classification rule which comes from the use of a different definition of neighborhood is introduced in this paper. The experimental results on Reuters-21578 standard benchmark collection show that our algorithm achieves better classification rates than the $k$-NN rule while decreasing classification time.
\end{abstract}

\section{Introduction}

Text Categorization (TC - also known as text classification) is the task of assigning documents to one or more predefined categories (or classes, or topics). This task relies on the availability of an initial corpus of classified documents under these categories (known as training data). Depending on the application, TC may be either single-label (i.e., exactly one category must be assigned to each document) or multi-label (i.e., any number of categories may be assigned to each document). This task, that falls at the crossroads of Information Retrieval and Machine Learning, has witnessed a booming interest in the last ten years from researchers and developers alike [1].

Text Categorization is an important component in many information management tasks such as spam filtering, real time sorting of email or files into folders, document routing, document dissemination, topic identification, classification of Web pages and automatic building of Yahoo!-style catalogs. Different learners have been applied in the TC literature, including probabilistic methods, decision tree and decision rule learners, example-based methods, support vector machines and classifier committees.

Nearest neighbor (NN) rule [2] is a very popular nonparametric and example-based method. This approach classifies an unknown sample into the categories of its nearest neighbors, according to some similarity measure. A particular case of $\mathrm{NN}$ classifiers is the $k$-nearest neighbor rule $(k-\mathrm{NN})$, which assigns the category most frequently represented among the $k$ nearest training samples. The NN classifiers include the following features: 1) conceptual simplicity, 2) easy implementation, 3) they can be designed even if training samples are few, 4) known error rate bounds, 5) they can be implemented when categories are overlapped with each other, 6) good performance, 7) they have no design phase and simply store the training set, and 8) they can be performed in time linear in the cardinality of the training set. 
During the last years, a large number of NN algorithms have aroused from various scientific communities. Many of them focus on reducing classification time [34]. Other algorithms focus on increasing classification rates, either changing the method to find nearest neighbors [5], varying the voting schema [6] or improving the training data [7].

NN classification rule has shown to be very effective in Text Categorization tasks. WAKNN [8] is a $k$-NN classification algorithm that learns importance of attributes and utilizes them in the similarity measure. Galavotti [6] proposed a variant of the basic $k$-NN approach based on the exploitation of the negative evidence. Yang [9] presents an examination of thresholding strategies on the performance of the $k$-NN classifiers under various conditions.

In this paper we introduce a new NN classification rule that obtains classification rates better than $k$-NN algorithm while decreasing classification time. This approach is based on a new definition of neighborhood. The effectiveness of the proposed algorithm is evaluated on different subsets of Reuters-21578 test collection, which is the standard benchmark for the Text Categorization tasks.

\section{A New Classification Rule}

As mentioned above, the nearest neighbor algorithms classify an unknown document $d$ into the categories of its nearest neighbors. These classifiers usually involve three phases: (i) the nearest neighbor finding from the training documents, (ii) a voting phase, in which each category assigns a vote to $d$, and (iii) a decision rule, in which a decision is made from these votes.

The first phase assumes a neighborhood definition. Intuitively, the concept of neighborhood should be such that the neighbors are as close to an unknown document $d$ as possible. The traditional $k$-nearest neighbor rule starts at $d$ and grows a spherical region until it encloses $k$ training documents. This kind of neighborhood suffers from some drawbacks: 1 ) the $k$ nearest neighbors may be too far from the document to classify $d$ (see Figure 1a), and 2) it may be not spatially homogeneous, that is, there are few neighbors very similar whereas others are not sufficiently close to $d$ (see Figure 1(b)), and hence the far documents can be decisive in the classification. The second one could occur, for example, when the training set is imbalanced (some categories are represented by a very small number of examples compared to the other categories).

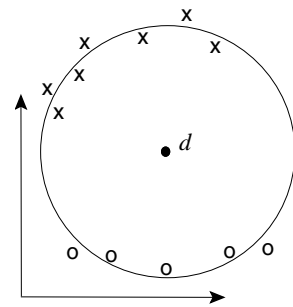

(a)

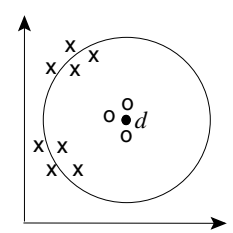

(b)

Fig. 1. Drawbacks of $k$-NN neighborhood. The big circles denote the "influence area" of the classifier. Here, we consider $k=7$. Note that, for ease of illustration, document similarities are here viewed in terms of Euclidean distance rather than in terms of cosine measure. 
In order to tackle these problems just described, we propose an alternative of neighborhood definition. The lower the neighborhood density of $d$, the greater the radius of spherical region defined by the $k$ nearest neighbors is. A natural way of avoiding that far documents influence in the classification is to fix a radius $\beta$ and take into account the training documents only whose similarities with $d$ are greater than or equal to $\beta$. Thus, documents which are not close to $d$ are excluded from the neighborhood.

However, this solution is not enough. A large value of $\beta$ may be very restrictive because many documents can not be classified. On the contrary, a small value of $\beta$ could cause many documents in the neighborhood, which may produce an important deterioration of the classification accuracy, in particular with documents belonging to the less represented categories. In order to focus on a sufficiently small neighborhood of training examples homogeneously distributed, we propose to automatically adjust the neighborhood radius from the nearest neighbor of $d$. With this aim, we define a spherical region, whose radius is the difference between the similarity of nearest neighbor of $d$ and a certain threshold $\alpha$. Thus, the neighborhood of the document to classify is defined by the intersection between this region and the spherical region of radius $\beta$. Notice that the conventional neighborhood takes into account the $k$ neighbors from the nearest neighbor. The neighborhood we propose takes into account instead all neighbors enclosed within a spherical region defined from the nearest neighbor. The parameters $\alpha$ and $\beta$ provide a convenient way of obtaining such a neighborhood.

The neighborhood definition proposed is graphically depicted in Figure 2 In the first case, the similarity ( $\max$ ) between the document to classify $d$ and its nearest neighbor is less than $\beta$; hence, the neighborhood is empty and the document can not be classified. The figures 2 (b) and (c) show the cases where $\beta<\max -\alpha$ and $\beta>\max -\alpha$, respectively. In both cases, the shady region represents the neighborhood of $d$.

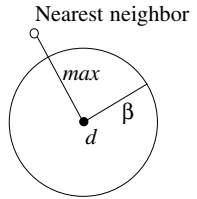

(a)

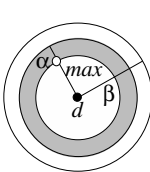

(b)

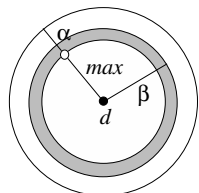

(c)

Fig. 2. Our neighborhood definition

Different methods have been used to calculate the votes of each category (second phase). In the basic $k$-NN classifier, the vote of the category $c_{i}$ is the number of nearest neighbors labeled with it. Another well-known method considers the similarity of the nearest neighbors and their category association to calculate the votes [10], i.e.,

$$
V\left(c_{i}, d\right)=\frac{\sum_{d_{j} \in N\left(c_{i}\right)} \cos \left(d, d_{j}\right)}{\sum_{d_{j} \in N} \cos \left(d, d_{j}\right)}
$$

where $N$ is the set of all nearest neighbors of the document $d, N\left(c_{i}\right)$ is the set of the nearest neighbors labeled with the category $c_{i}$, and $\cos \left(d, d_{j}\right)$ is the cosine between the 
two document vectors, which is the similarity measure commonly used in TC tasks. In this paper, we used the second method.

From these category votes, several rules can be applied for deciding whether $d$ should be classified under $c_{i}$ (third phase). A simple decision rule classifies the document into the category that has the greatest vote. Instead, we used the thresholding decision rule. According with this rule, the document is assigned to categories with the score greater than a certain threshold value $\gamma$. Notice that this decision rule allows a multi-label categorization. There are various policies for determining the thresholds [9]. We adopt the policy $C S V$ thresholding, which consists in testing different values on a validation set and choosing the value which maximizes effectiveness (we use $\gamma=0.3$ ).

To sum up, the steps of our NN classifier is shown in Algorithm 1

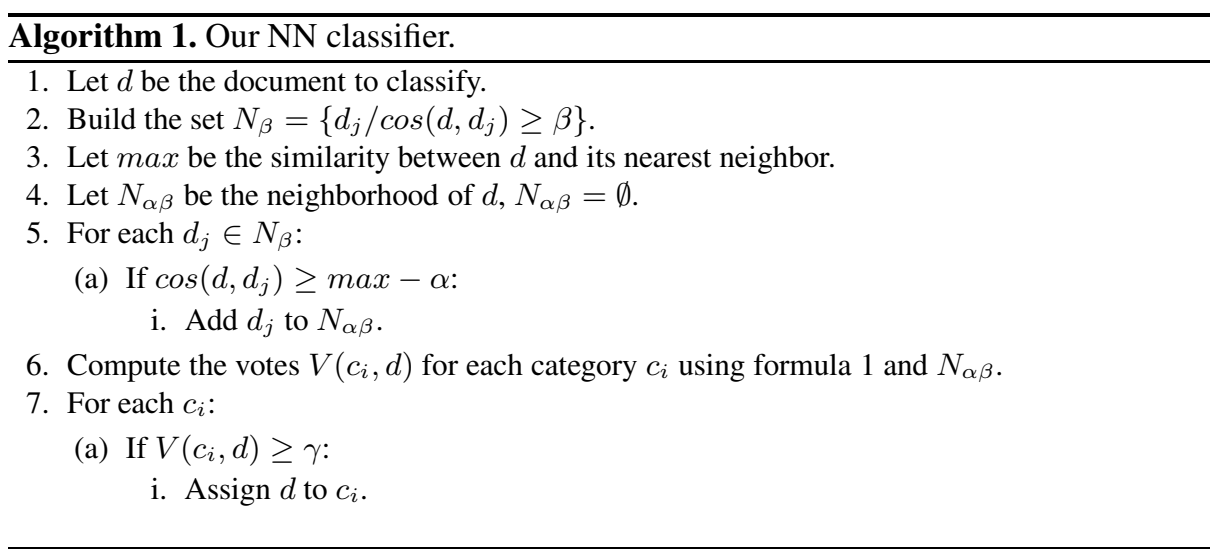

Like $k$-NN, in our algorithm the classification of a document is performed in linear time with respect to the cardinality of the training set.

\section{Experiments}

We have conducted some experiments to test the validity of our NN rule for Text Categorization tasks.

\subsection{Document Indexing}

Because of texts cannot be directly interpreted by a classifier, an indexing procedure that maps a document into a compact representation of its content needs to be applied. In this paper, we adopt the traditional vector space model, in which a document $d_{j}$ is represented as a vector of term weights $d_{j}=\left(w_{1}^{j}, \ldots, w_{|\tau|}^{j}\right)$, where $\tau$ is the set of terms that occur in the document collection and quantifies the importance of term $t_{l}$ in characterizing the semantics of $d_{j}$. The selection of terms includes removing tags and stop words, lemmatization and proper name recognition. Weights are computed by using the standard ltc variant of $t f$-idf function [11], i.e., $w_{l}^{j}=\left(1+\log T F\left(t_{l}, d_{j}\right)\right)$. 
$\log \frac{|T r|}{d f_{T r}\left(t_{l}\right)}$, where $T F\left(t_{l}, d_{j}\right)$ denotes the number of times $t_{l}$ occurs in $d_{j}, T r$ is the set of training documents and $d f_{T r}\left(t_{l}\right)$ is the number of documents in $\operatorname{Tr}$ in which $t_{l}$ occurs at least once.

In TC, unlike in Information Retrieval, a dimensionality reduction phase is often applied so as to reduce the size of the document representations from $|\tau|$ to a much smaller, predefined number $\left|\tau^{\prime}\right|$; the value $\varepsilon=\frac{|\tau|-\left|\tau^{\prime}\right|}{|\tau|}$ is called the reduction factor [12]. Dimensionality reduction techniques reduce overfitting (i.e., the tendency of the classifier to better classify the data it has been trained on than new unseen data), and makes the problem more manageable for the classifier. Also, standard classification techniques cannot deal with a large feature set, since processing is extremely costly in computational terms, and the results become unreliable due to the lack of sufficient training data.

Usually, these techniques consist in scoring each term in $\tau$ by means of a categorybased term evaluation function $f$ and then selecting a set $\tau^{\prime}$ of the highest scoring terms. The information gain [13] is a standard function of the trade in the dimensionality reduction literature, i.e.,

$$
f\left(t_{l}, c_{i}\right)=\sum_{c \in\left\{c_{i}, \overline{c_{i}}\right\}} \sum_{t \in\left\{t_{l}, \overline{t_{l}}\right\}} P(t, c) \cdot \log _{2} \frac{P(t, c)}{P(t) \cdot P(c)}
$$

In this formula, probabilities are interpreted on an event space of documents (e.g. $P\left(\bar{t}_{l}, c_{i}\right)$ indicates the probability that, for a random document $d$, term $t_{l}$ does not occur in $d$ and $d$ belongs to category $c_{i}$ ), and are estimated by maximum likelihood. We use this function to select terms according to the maximum global policy, i.e.,

$$
f_{\max }=\max _{i=1} f\left(t_{l}, c_{i}\right)
$$

\subsection{Experimental Settings}

In our experiments we have used the Reuters-21578, Distribution 1.0 corpus 1 , currently the most widely used benchmark in TC research. Reuters-21578 consists of a set of 12902 news stories classified under 135 categories related to economics. This collection is partitioned (according to the "ModApté" split we have adopted) into a training set of 9603 documents and a test set of 3299 documents.

Several researchers have preferred to carry out their experiments on different subsets of categories. The three subsets that have been most popular [12] are: 1) the set of the 10 categories with the highest number of positive training examples, hereafter, reu10, 2 ) the set of 90 categories with at least one positive training example and one test example, hereafter, reu90, and 3) the set of 115 categories with at least one positive training example, hereafter, reu115. The characteristics of these subsets are summarized in Table 1. The last four columns contain the average number of categories per document, and the maximum, minimum and average number of training examples per category, respectively.

\footnotetext{
${ }^{1}$ The Reuters-21578 collection may be freely download from http://kdd.ics.uci.edu.
} 
Table 1. Characteristics of Reuters-21578 subsets

\begin{tabular}{|c|c|c|c|c|c|c|}
\hline Collection & \# Train Docs. & \# Test Docs. & Multi-label & \multicolumn{3}{|c|}{ Category size } \\
\cline { 4 - 7 } & & & & Max & Min & Average \\
\hline \hline reu10 & 5920 & 2313 & 1.11 & 2709 & 160 & 657 \\
\hline reu90 & 7058 & 2740 & 1.24 & 2709 & 1 & 98 \\
\hline reu115 & 7063 & 2740 & 1.25 & 2709 & 1 & 76 \\
\hline
\end{tabular}

TC effectiveness is usually measured in terms of the classic Information Retrieval notions of precision $\left(\pi_{i}\right)$, the proportion of documents classified into $c_{i}$ that indeed belong to $c_{i}$, and recall $\left(\rho_{i}\right)$, the proportion of documents belonging to $c_{i}$ that are indeed classified into $c_{i}$. Since a classifier can be tuned to emphasize precision at the expense of recall, or vice versa, only combinations of the two are significant. The most popular combination nowadays is: $F 1\left(c_{i}\right)=\frac{2 \pi_{i} \rho_{i}}{\pi_{i}+\rho_{i}}=\frac{2 T P_{i}}{2 T P_{i}+F P_{i}+F N_{i}}$, where $T P_{i}, F P_{i}$ and $F N_{i}$ refer to the sets of true positives wrt $c_{i}$ (documents correctly deemed to belong to $c_{i}$ ), false positives wrt $c_{i}$ (documents incorrectly deemed to belong to $c_{i}$ ), and false negatives wrt $c_{i}$ (documents incorrectly deemed not to belong to $c_{i}$ ), respectively.

When effectiveness is computed for several categories, the results for individual categories must be averaged in some way. Two methods may be adopted: micro-averaging (categories count proportionally to the number of their positive test examples) and macro-averaging (all categories count the same). Micro-averaging gives equal weight to every document, while macro-averaging gives equal weight to each category. The former rewards classifiers that behave well on heavily populated ("frequent") categories, while classifiers that perform well also on infrequent categories are emphasized by the latter.

\subsection{Results}

The first experiments we have conducted compare the categorization performance of our NN rule against $k$-NN classifier on the three above-mentioned Reuters-21578 subsets. Both approaches use the same method to calculate the votes of each category and the thresholding decision rule.

Different values of parameters have been tried on each algorithm to ensure that the experimental results can reflect the best performance. The values of $k$ tried for the $k$ NN algorithm include 1, 3, 5, 7, 10, 20, 30, 40 and 50. There are two parameters of importance in our NN algorithm: $\alpha$ and $\beta$. The values of $\alpha$ tried are $0.1,0.15,0.20$, 0.25 and 0.3 . However, the value of $\beta$ is fixed to 0.1 , since its role is only to guarantee that far documents do not influence in the classification. Then, we chose the parameters with the best performance according to micro-averaged $F 1$ to represent each algorithm.

The results of our experiments are reported in Figure 3 This figure includes 6 plots: the leftmost plots show micro-averaged $F 1$ scores while the rightmost show macroaveraged $F 1$ scores. Each individual plot, which corresponds to a dimensionality reduction factor, includes the results for both NN approaches in each document collection.

Several observations can be made by analyzing the results in Figure 3. First, the best performance is obtained in Reu10 subset for both algorithms. This was largely to be expected, given that its categories are the ones with the highest number of training 

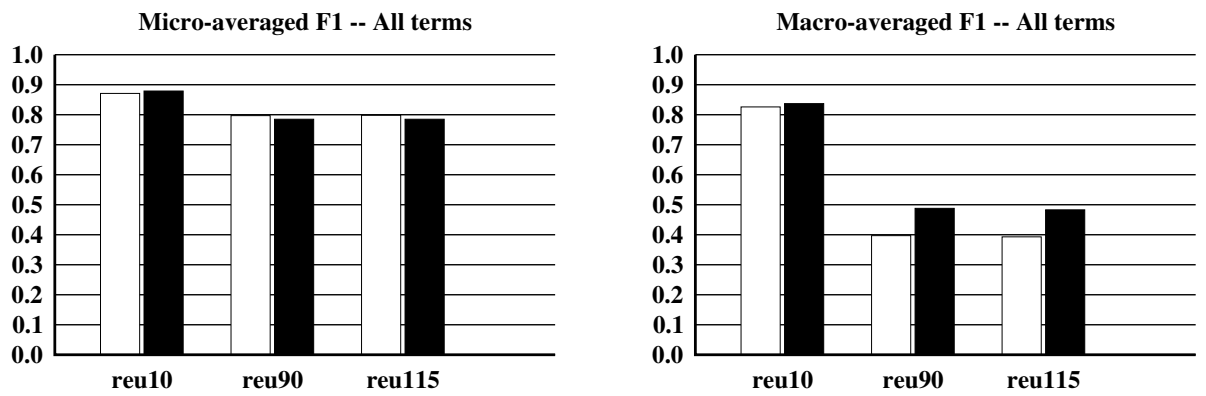

Micro-averaged F1 -- Reduction factor $=0.5$

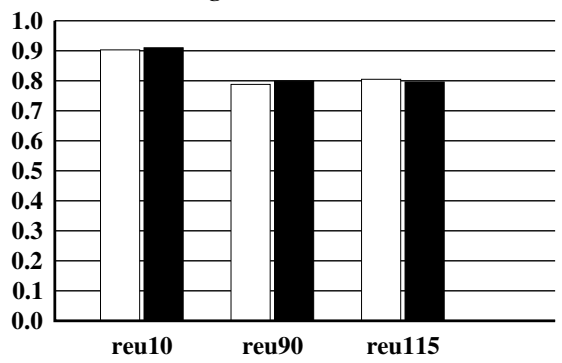

Micro-averaged F1 -- Reduction factor=0.9
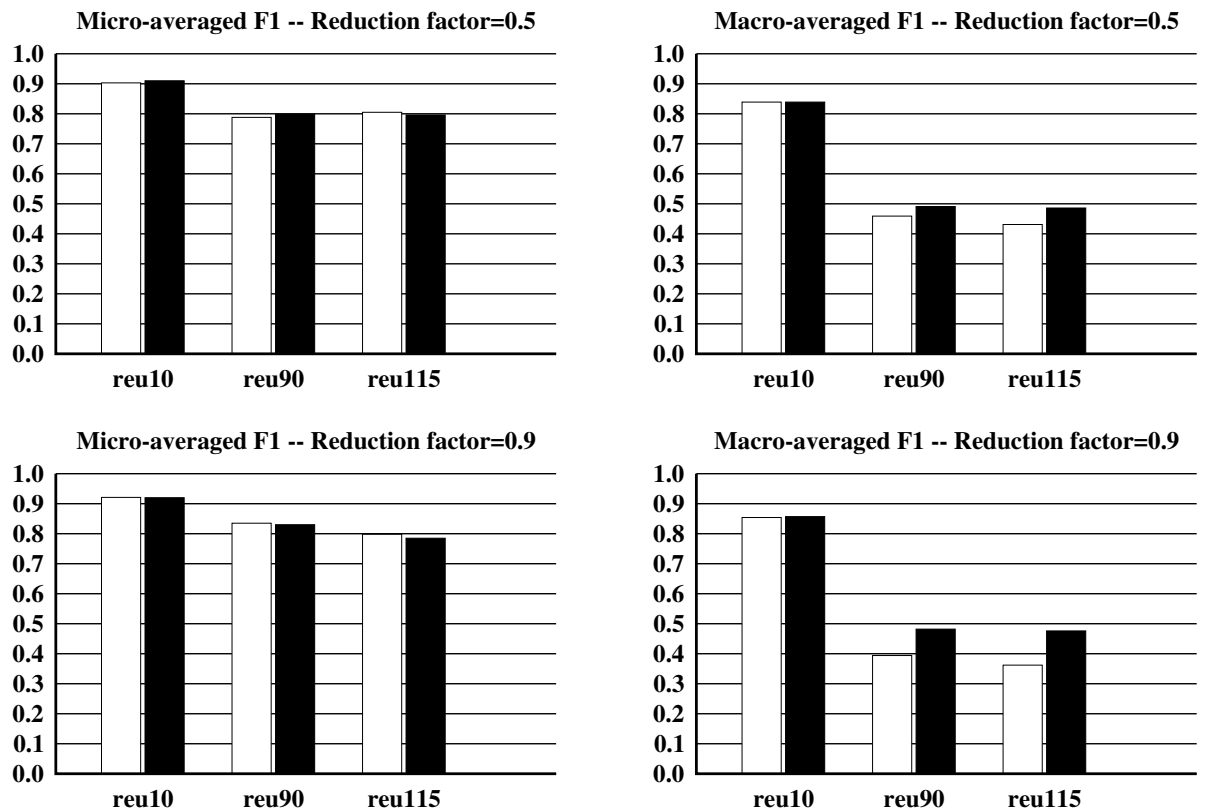

Macro-averaged F1 -- Reduction factor=0.9

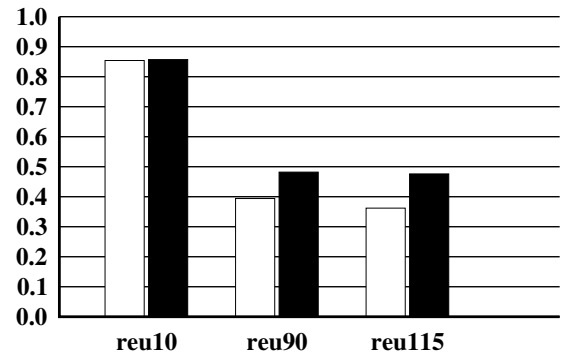

Fig. 3. $F 1$ scores obtained with $k$-NN (white bars) and our NN rule (black bars)

examples, and as such allow taming the "curse of dimensionality" more effectively. The decrease of performance in going from Reu10 to Reu90 is much larger for macroaveraged $F 1$ than micro-averaged $F 1$. However, the performances of Reu 90 and Reu 115 have a similar behavior. This can be explained by the fact that micro-averaged effectiveness is dominated by the performance of the classifiers on the most frequent categories. Note that the 10 categories in Reu10 have altogether 2313 test examples, while the other 80 categories in Reu90 have altogether just 427 them. Also, the differences between Reu90 and Reu115 are limited to 5 documents and 25 very small categories. These conclusions are in agreement with the results presented earlier in [12].

A second fact that also emerges clearly from the figure 3 is that the best microaveraged $F 1$ scores are achieved with the most aggressive reduction in all collections. Macro-averaged effectiveness maintains this behavior in Reu10 subset. Instead, in the other subsets the behavior of the algorithms is different. Our NN rule obtains the same 


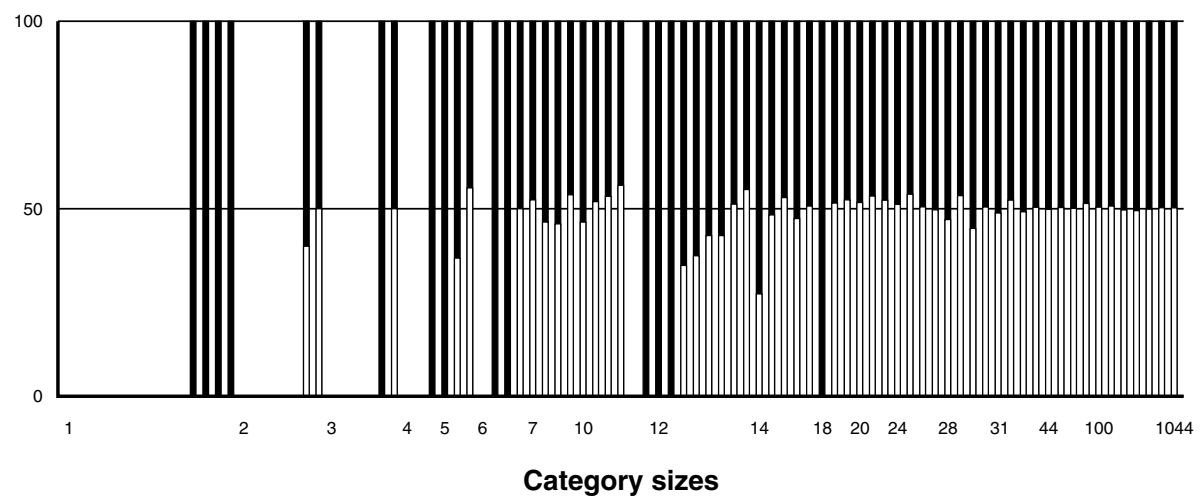

Fig. 4. $F 1$ scores per category with $k$-NN (white bars) and our NN rule (black bars)
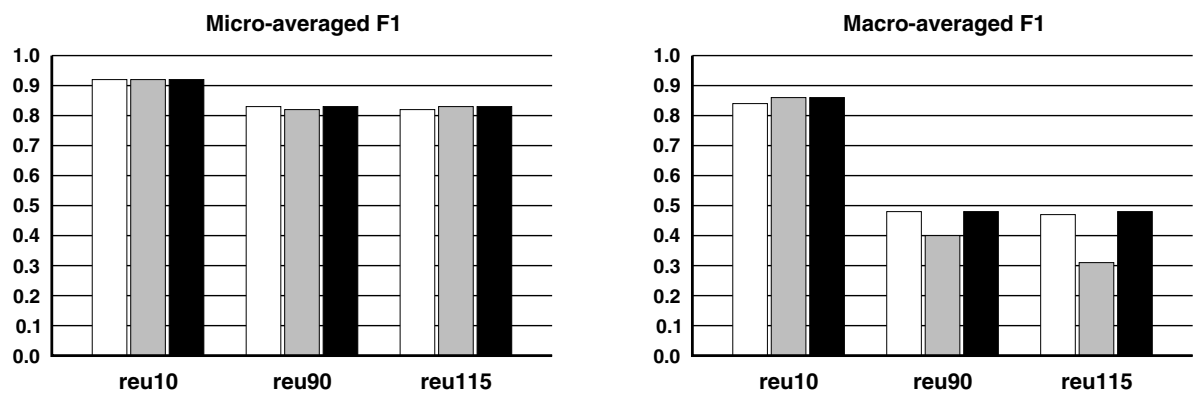

Fig. 5. Impact of the parameters in our $\mathrm{NN}$ rule

macro-averaged $F 1$ score disregarding the reduction factor whereas $k$-NN improves effectiveness when term reduction is $50 \%$, but decreases it with $90 \%$ of reduction.

Finally, the micro-averaged $F 1$ scores obtained by both algorithms are similar in all collections. However, with regard to the macro-averaged $F 1$ our NN rule clearly overcomes $k$-NN in Reu90 and Reu115 subsets. This observation seems to suggest that our algorithm yields better results when categories have few training examples. In order to know what really happens, Figure 4 shows a percentage diagram of $F 1$ scores per category in Reu90 collection with $90 \%$ of reduction factor.

As it can be noticed, both algorithms obtain similar results for the most frequent categories. Nevertheless, our approach achieves better results than $k$-NN when categories have few training examples. This can be explained because the best results in $k$-NN algorithm are obtained with $k=30$, and hence it is difficult that small categories achieve the required votes. Since the number of neighbors in our algorithm is not fixed, this problem is reduced. It must be pointed out that there are still some less represented categories whose documents are not well-classified.

Our second experiment was focused on evaluating the impact of the parameters $\alpha$ and $\beta$ in the classification rates. Figure 5 shows the $F 1$ scores obtained when disregarding the $\beta$ parameter (white bars), disregarding the $\alpha$ parameter (gray bars) and considering 


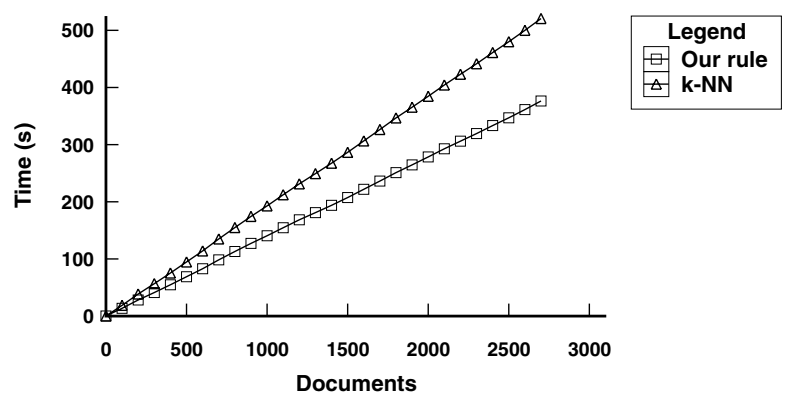

Fig. 6. Time performance

both parameters (black bars). As it can be noticed, the $F 1$ scores are similar when we only consider the $\alpha$ parameter and both parameters together. Despite these results, we believe that $\beta$ parameter is useful to avoid that far documents influence in the classification, although it seems that this problem is not presented in the Reuter collection.

Finally, Figure 6 shows the time spent by $k$-NN and our algorithm. As can be seen, despite both algorithms have a linear behavior, our algorithm clearly overcomes $k$-NN. This can be explained because we do not need to create a sorted list of $k$ nearest neighbors.

\section{Conclusions}

A new NN classification rule has been proposed in this paper. It is an attempt to minimize the drawbacks pointed out for the $k$-NN rule. This approach is based on the general idea of estimating the category of an unknown document $d$ from its neighbors, but considering a kind of neighborhood which allows one to inspect a sufficiently small and near area to $d$. In our rule the number of neighbors is not fixed, but rather the neighborhood radius is automatically adjusted from the nearest neighbor of $d$.

The experiments carried out on Reuters-21578 standard benchmark collection show that our rule not only obtains similar micro-averaged $F 1$ scores, but it also achieves better macro-averaged $F 1$ scores than $k$-NN. It is very important since producing classifiers that perform well also on infrequent categories is the most challenging problem of Text Categorization [1]. Furthermore, our algorithm reduces the classification time.

In the future, one key issue we face is the automatic choice of the threshold value $\alpha$. Future work also includes exploring the use of our NN rule in adaptive document filtering tasks, where the category distribution is not given a priori and it must be learned incrementally over time.

\section{References}

1. Sebastiani, F.: Text categorization. In: Text Mining and its Applications to Intelligence, CRM and Knowledge Management. WIT Press, Southampton, UK (2005)

2. Duda, R., Hart, P., Stark, D.G.: Pattern Classification. Wiley-Interscience (2000) 
3. Ramasubramanian, V., Paliwal, K.K.: Fast nearest-neighbor search algorithms based on approximation-elimination search. Pattern Recognition 33 (2000) 1497-1510

4. Moreno-Seco, F., Micó, L., Oncina, J.: A new classification rule based on nearest neighbour search. In: 17th International Conference on Pattern Recognition. Volume 4., IEEE Computer Society (2004) 408-411

5. Sánchez, J., Pla, F., Ferri, F.: On the use of neighbourhood-based non-parametric classifiers,. Pattern Recognition Letters 18 (1997) 1179-1186

6. Galavotti, L., Sebastiani, F., Simi, M.: Experiments on the use of feature selection and negative evidence in automated text categorization. In: ECDL-00, 4th European Conference on Research and Advanced Technology for Digital Libraries, Lisboa, PT (2000) 59-68

7. Shin, K., Abraham, A., Han, S.: Improving knn text categorization by removing outliers from training set. In: 7th International Conference of Computational Linguistics and Intelligent Text Processing,CICLing 2006. (2006) 563-566

8. Han, E.H., Karypis, G., Kumar, V.: Text categorization using weight adjusted k-nearest neighbor classification. In: 5th Pacific-Asia Conference on Knowledge Discovery and Data Mining (PAKDD). (2001) 53-65

9. Yang, Y.: A study on thresholding strategies for text categorization. In: SIGIR-01, 24th ACM International Conference on Research and Development in Information Retrieval, New York, ACM Press (2001) 137-145

10. Yang, Y.: Expert network: Effective and efficient learning from human decisions in text categorization and retrieval. In: SIGIR'94,17th ACM International Conference on Research and Development in Information Retrieval, Ireland (1994) 13-22

11. Salton, G., Buckley, C.: Term-weighting approaches in automatic text retrieval. Information Processing and Management 24 (1988) 513-523

12. Debole, F., Sebastiani, F.: An analysis of the relative hardness of Reuters-21578 subsets. Journal of the American Society for Information Science and Technology 56 (2005) 584596

13. Lewis, D.D.: An evaluation of phrasal and clustered representations on a text categorization task. In: SIGIR-92, 15th ACM International Conference on Research and Development in Information Retrieval, Denmark (1992) 37-50 